\title{
Malignant Uterine Corpus Neoplasm
}

National Cancer Institute

\section{Source}

National Cancer Institute. Malignant Uterine Corpus Neoplasm. NCI Thesaurus. Code C3556.

A malignant neoplasm that affects the uterine corpus. Representative examples include endometrial carcinoma, carcinosarcoma, leiomyosarcoma, and adenosarcoma. 